\title{
Uso de gentamicina transtimpánica: Experiencia del Hospital Clínico de la Universidad de Chile Transtympanic gentamicin: Experience in Hospital Clínico Universidad de Chile
}

\author{
Karen García C', Pablo Cantillano $\mathbf{P}^{1}$, Natalia Cabrera $S^{2}$, Paul Délano $\mathbf{R}^{1,3}$.
}

\begin{abstract}
RESUMEN
Introducción: La gentamicina transtimpánica se utiliza en el manejo de pacientes con vértigo crónico refractario al manejo médico.

objetivo: Describir y analizar las características clínicas, epidemiológicas, y resultados de pacientes sometidos a tratamiento con gentamicina transtimpánica en el Hospital Clínico de la Universidad de Chile.

Material y método: Estudio retrospectivo, descriptivo, incluyendo los pacientes con patología otorrinolaringológica que hayan recibido gentamicina transtimpánica entre los años 2008 y 2018. Se analizaron variables epidemiológicas, clínicas, y función vestibular.

Resultados: La serie está constituida por diez pacientes, con una edad promedio de 52,4 años; siete mujeres y tres hombres. El diagnóstico fue en su mayoría enfermedad de Ménière (7 pacientes). El número promedio de inyecciones de gentamicina fue de 2,8. En el período de seguimiento (rango 1-96 meses), se logró mejoría del vértigo en la mayoría de los pacientes (8 de 10).

Conclusión: El tratamiento con inyecciones de gentamicina transtimpánica es una opción importante para aliviar la sintomatología de pacientes con vértigo crónico intratable. Debe indicarse en casos seleccionados, y siempre realizar un estudio auditivo y vestibular completo antes y después del tratamiento con gentamicina.

Palabras clave: Vértigo, mareos, gentamicina, enfermedad de Ménière, hídrops endolinfático.
\end{abstract}

\begin{abstract}
Introduction: Transtympanic gentamicin is used for treatment of patients with chronic vertigo refractory to medical management.
\end{abstract}

\footnotetext{
1 Departamento de Otorrinolaringología, Hospital Clínico Universidad de Chile, Santiago, Chile.

2 Servicio de Otorrinolaringología, Hospital El Carmen, Maipú, Santiago, Chile.

3 Departamento de Neurociencia, Facultad de Medicina, Universidad de Chile, Santiago, Chile.
}

Los autores declaran no tener conflictos de interés. P.D.R. es financiado por CONICYT BASAL FB008, Proyecto ICM P09-015F y Fundación Guillermo Puelma.

Recibido el 11 de octubre, 2018. Aceptado el 6 de mayo, 2019. 
Aim: To describe and analyze the clinical outcome of patients whom underwent treatment with transtympanic gentamicin at the Clinical Hospital Universidad de Chile.

Material and method: Retrospective and descriptive study including patients with otolaryngologist disease whom underwent treatment with transtympanic gentamicin between 2008 and 2018. Epidemiological, clinical variables and vestibular function were analyzed.

Results: The serie consists of ten patients, 7 men and 3 women, with an average age of 52,4 years. The most frequent diagnosis was Ménière disease (7 patients). The average number of gentamicin injections was 2,8. The follow up varies from 1 to 96 months, presenting improvement of vertigo in the majority of the cases (8 of 10 patients).

Conclusion: The treatment with transtympanic injections of gentamicin is a relevant option to decrease symptoms in patients with chronic intractable vertigo. It should be indicated in selected patients. Prior and posterior the treatment, patients must be studied with a complete evaluation of the auditory and vestibular function.

Key words: Vertigo, dizziness, gentamicin, Ménière disease, endolymphatic hydrops.

\section{INTRODUCCIÓN}

La gentamicina es un antibiótico de la familia de los aminoglicósidos con propiedades ototóxicas, preferentemente para las células ciliadas de los receptores vestibulares. De esta forma, se propone la administración de gentamicina como terapia ablativa de la función vestibular en pacientes con vértigo crónico refractario al manejo médico habitual. La gentamicina puede ser colocada por un médico otorrinolaringólogo en la cavidad del oído medio a través de inyecciones transtimpánicas. Dada la permeabilidad de la ventana redonda de la cóclea, al depositar gentamicina en el oído medio, ésta es transportada al oído interno, donde ejerce su función ototóxica, especialmente en las células de los epitelios vestibulares. La gran mayoría de los pacientes a los que se les indica gentamicina transtimpánica (GTT) tienen diagnóstico de enfermedad de Ménière (EM) 0 hídrops endolinfático secundario.

\section{OBJETIVO}

Describir y analizar las características clínicas, epidemiológicas, y los resultados sintomáticos, vestibulares y audiológicos del tratamiento con GTT de los pacientes que hayan sido evaluados en el Servicio de Otorrinolaringología del Hospital Clínico de la Universidad de Chile ( $\mathrm{HCUCH}$ ) entre los años 2008 y 2018.

\section{MATERIAL Y MÉTODO}

Se realizó un estudio retrospectivo, descriptivo, de todos los pacientes que hayan recibido GTT, entre los años 2008 y 2018, en el HCUCH. Se revisaron las fichas clínicas para obtener datos epidemiológicos, clínicos, y evaluaciones auditivas y vestibulares antes y después de la GTT. Todos los pacientes disponen de estudio secundario de hidropesía endolinfática incluyendo resonancia magnética de cerebro, exámenes treponémicos y no treponémicos para sífilis, perfil reumatológico y función tiroidea entre otros. Todos los pacientes cuentan con audiometría y estudio vestibular con prueba calórica con video-oculografía (VOG) y/o prueba de impulso cefálico asistida por video y acelerómetro (vHIT por sus siglas en inglés) antes del tratamiento con GTT.

Se utilizaron ampollas de $80 \mathrm{mg}$ de gentamicina en $2 \mathrm{ml}$, inyectando un volumen cercano a los 0,4 ml en la cavidad del oído medio. Se realizó la inyección bajo microscopio y técnica estéril en el cuadrante antero-inferior, con trócar lumbar №25, previa aplicación de lidocaína $4 \%$ en forma tópica. Los pacientes se mantuvieron en reposo en decúbito supino con cabeza rotada en $45^{\circ}$ hacia el lado contralateral por al menos 20 minutos. Se les pidió no tragar ni hablar en este tiempo. Posterior al inicio de la terapia ablativa, todos los pacientes siguieron un protocolo de rehabilitación vestibular que se inició en los primeros días posteriores a la GTT, por tecnólogo médico del Servicio de Otorri- 
nolaringología del $\mathrm{HCUCH}$. Este trabajo cuenta con la aprobación del Comité de Ética del $\mathrm{HCUCH}$.

\section{RESULTADOS}

\section{Demográfica}

La serie de casos clínicos está constituida por diez pacientes; siete mujeres y tres hombres. La edad promedio de intervención fue de 52,4 años (rango 43-56 años). Los datos de los pacientes se resumen en la Tabla 1. El diagnóstico que motivó la indicación de GTT fue en su mayoría por EM (7 casos). Además, se incluyen casos aislados de hídrops endolinfático autoinmune, hídrops retardado y fístula perilinfática. La lateralidad fue en oído izquierdo en ocho pacientes, y oído derecho en dos pacientes. Todos los pacientes recibieron tratamiento médico previo, incluyendo evaluación por psiquiatría, sin respuesta sintomática. El tratamiento farmacológico incluyó diversos antivertiginosos (difenidol, dimenhidrato, y betahistina) y en tres casos, inyecciones de corticoide transtimpánico. El promedio de dosis de GTT fue de 2,8 dosis, con un rango de 1 a 6 dosis, separadas por al menos un mes.

\section{Hallazgos clínicos}

Todos los pacientes presentaban vértigo intratable previo a la GTT, un paciente presentó crisis otolítica de Tumarkin. Previo y posterior a la administración de GTT se realizó estudio con VIII par con VOG. EI registro de estos datos es completo para 5 pacientes (casos 2, 3, 5, 6 y 9 de la Tabla 1), sobre los cuales se presentan los siguientes resultados en la Tabla 2. El promedio tonal puro de la vía aérea $(0,5-2 \mathrm{kHz})$ antes y después del tratamiento de GTT fue de 56 $\mathrm{dB} H \mathrm{HL}$ y $62,2 \mathrm{~dB} H \mathrm{HL}$ respectivamente, mientras que la discriminación promedio de disílabos, previo al tratamiento fue de $72,8 \%$, y posterior a éste; $72,8 \%$. En cuanto al compromiso vestibular: el promedio de hipoexcitabilidad en el oído afectado, medido con la velocidad de la componente lenta de la prueba calórica fue de $25,9 \%$ y $34,5 \%$ antes y después de la GTT respectivamente. En la Figura 1 se muestra como ejemplo, al caso 6 de la Tabla 1, quien se estudió con vHIT previo y posterior al tratamiento.

\section{Recurrencia}

El tiempo de seguimiento para esta serie, varía de 1 mes a 96 meses, presentando mejoría clínica sintomática en 8 pacientes. Uno de los diez pacientes, que recibió 6 inyecciones de gentamicina (paciente 4, Tabla 1) debió ser sometido a laberintectomía quirúrgica, con resolución del vértigo. Un segundo paciente, cuyo diagnóstico fue de fístula perilinfática posterior a timpanoplastía, recibió gentamicina directamente sobre la ventana redonda en pabellón, por recomendación del comité de oídos del Servicio de Otorrinolaringología del $\mathrm{HCUCH}$.

Tabla 1. Características epidemiológicas y clínicas de pacientes

\begin{tabular}{|c|c|c|c|c|c|c|c|}
\hline No & Edad & Sexo & Diagnóstico & Lateralidad & GTT & Seguimiento & Vértigo \\
\hline 1 & 48 & M & EM & 이 & 4 & 2 años & - \\
\hline 2 & 43 & M & EM & 이 & 4 & 8 años & - \\
\hline 3 & 52 & M & EM & OD & 6 & 3 años & - \\
\hline 4 & 48 & $\mathrm{~F}$ & $\mathrm{HAl}$ & 이 & 6 & $1 \mathrm{mes}$ & +, laberintectomía quirúrgica \\
\hline 5 & 50 & $\mathrm{~F}$ & EM & 이 & 1 & 10 meses & - \\
\hline 6 & 58 & $\mathrm{~F}$ & EM & 이 & 1 & 4 meses & - \\
\hline 7 & 56 & $\mathrm{~F}$ & $H R$ & 이 & 1 & 2 mes & * \\
\hline 8 & 56 & $\mathrm{~F}$ & FPL & 이 & 3 & 2 meses & +, laberintectomía química en pabellón \\
\hline 9 & 55 & $\mathrm{~F}$ & EM & OD & 1 & 2 años & - \\
\hline 10 & 58 & $\mathrm{~F}$ & EM & 이 & 1 & 1 mes & - \\
\hline
\end{tabular}

Edad en años, $\mathrm{M}$ =masculino, $\mathrm{F}$ =femenino, EM =Enfermedad de Ménière, $\mathrm{HAl}=$ hídrops autoinmune, $\mathrm{HR}$ =hídrops retardado, FPL =fístula perilinfática, OI =oído izquierdo, OD =oído derecho, GTT =número de dosis de gentamicina transtimpánica aplicadas. - =mejoría absoluta, * =mejoría sustancial, en base a sintomatología descrita por paciente. 


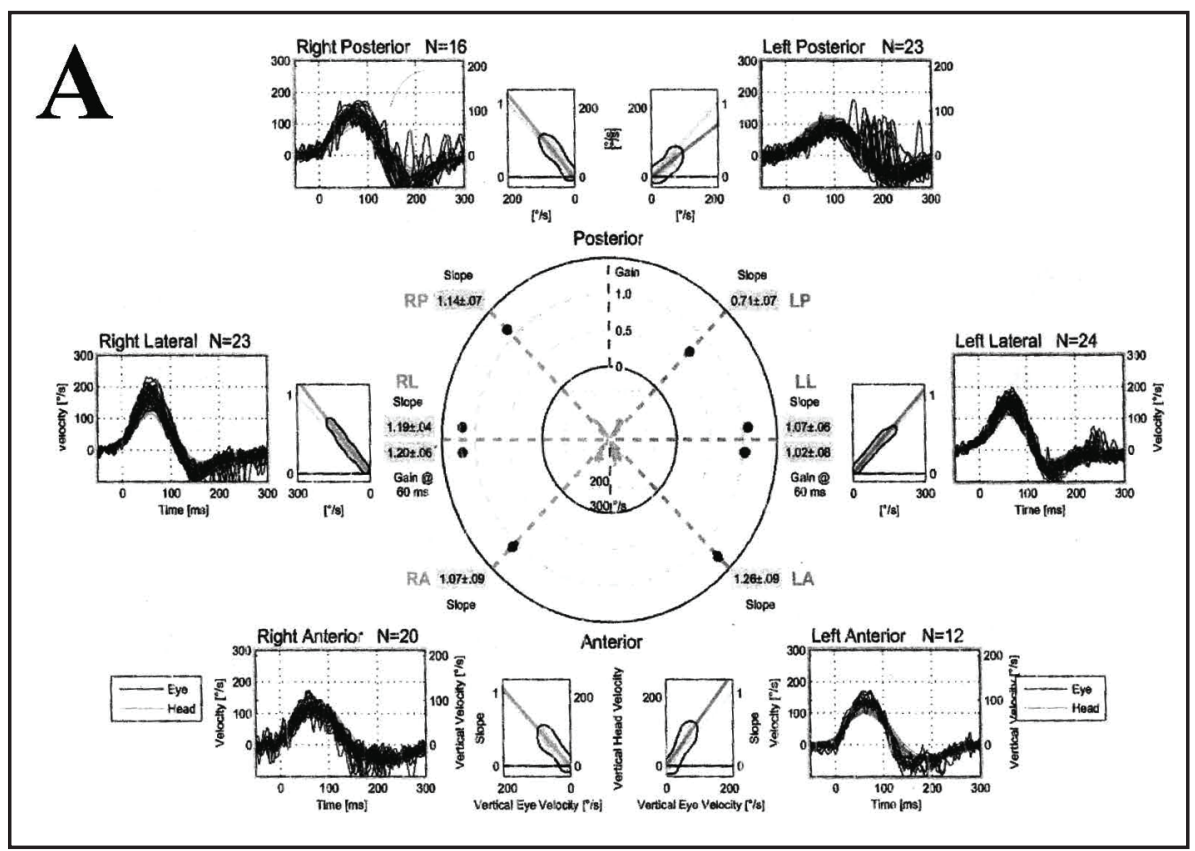

\section{B}

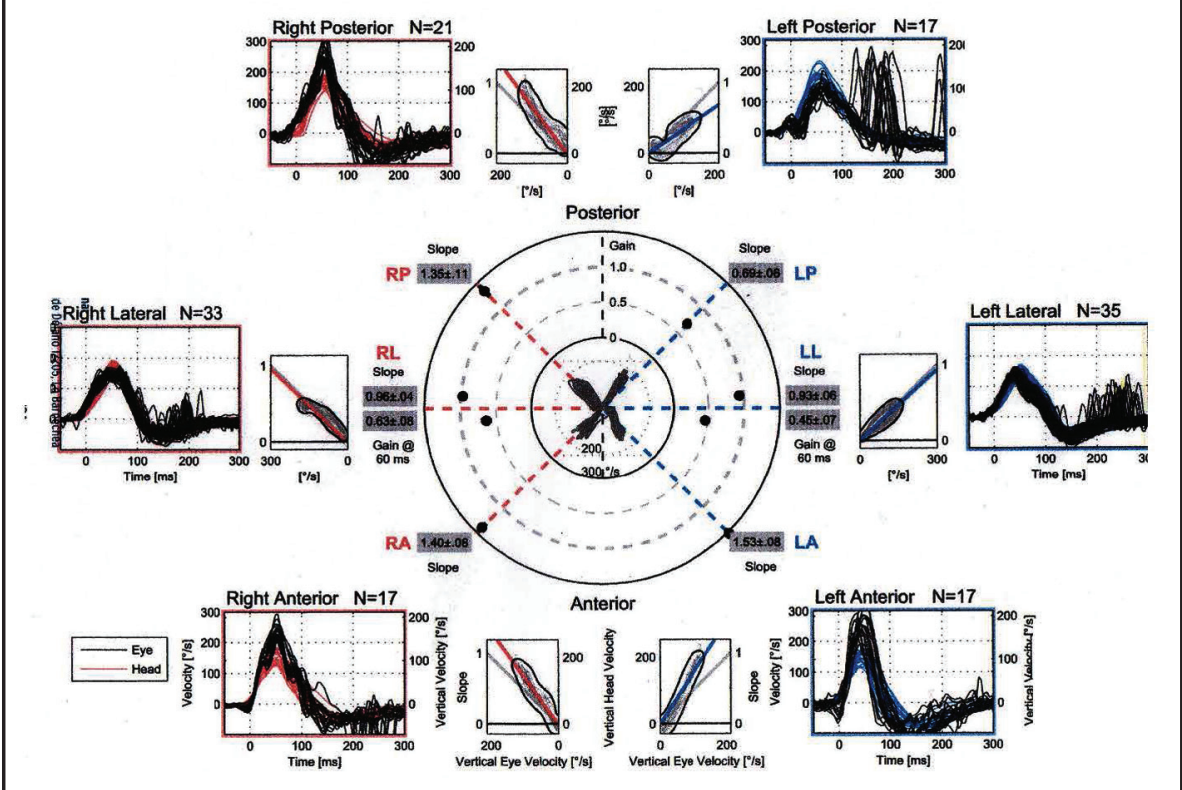

Figura 1. vHIT previo (A) y posterior (B) a gentamicina transtimpánica en el 0 l del paciente $\mathrm{N}^{0} 7$. 


\section{DISCUSIÓN}

\section{Farmacología}

Harold Schuknecht en 1956, fue el primero en administrar aminoglicósidos transtimpánicos en pacientes con EM, con buen control del vértigo, pero con desarrollo de hipoacusia sensorioneural profunda en todos los casos ${ }^{1}$. La histopatología en chinchillas muestra que el inicio del efecto de gentamicina en el oído interno sería alrededor de seis horas después de su administración en el oído medio. Mientras que después de 32 horas es posible encontrar daño evidente en las células ciliadas del oído interno². Los trabajos en modelos animales han mostrado que la toxicidad es mayor para las células receptoras vestibulares, en comparación a las cocleares, en particular en las células ciliadas tipo $\mathrm{I}^{3-5}$.

Es importante destacar que una semana posterior a la aplicación de gentamicina, ésta es aún detectable en el oído interno y que la administración por esta vía, es segura para el oído contralateral ${ }^{6}$. La ausencia de toxicidad en el oído contralateral al aplicar GTT es fundamental ya que la función auditiva remanente del oído contralateral es de vital importancia para mantener la comunicación por vía oral de los pacientes. Esto lo confirmamos en nuestros pacientes, que no presentaron toxicidad auditiva contralateral.

En relación al modo de administración, la GTT en humanos se ha utilizado disuelta en suero fisiológico $0^{7,8}$ y tamponada con bicarbonato de sodio $\left(\mathrm{NaHCO}_{3}\right)^{8-11}$, sin embargo, las últimas recomendaciones sugieren que no es necesario alcalinizar la solución de gentamicina y que es posible inyectarla directamente. Acorde a estas sugerencias, en nuestros casos clínicos, se inyectó GTT directamente sin disolver en bicarbonato con buena tolerancia y con buenos resultados clínicos.

\section{Efectividad de la GTT}

Un trabajo anterior describió la experiencia en Chile con terapia transtimpánica en EM con fracaso a terapia médica, incluyendo 6 pacientes que recibieron GTT en primera instancia, y 4 pacientes que recibieron GTT posterior a fracaso con corticoides transtimpánicos. En ambos casos, hubo mejoría de la sintomatología vestibular, según encuestatelefónica diseñada por los investigadores? ${ }^{7}$. También se había reportado un caso clínico de EM que requirió $\mathrm{GTT}^{12}$. Mientras que, en la literatura internacional, un metaanálisis de 2004, incluyó 14 estudios, con un total de 627 pacientes con EM, con GTT como terapia única, ya sea en forma de inyección 0 catéter. En su análisis, encontraron una mejoría completa del vértigo del $74,7 \%$, y de 92,7\% al incluir también aquellos pacientes con mejoría sustancial. Posterior al tratamiento con GTT, la audición disminuyó en forma global en 1,5 $\mathrm{dB}$, mientras que la discriminación, lo hizo en $2 \%$; ambos valores no estadísticamente significativos. Destaca que solo 7 estudios incluyeron el uso de prueba calórica, pero dada la variabilidad en la forma de exponer los resultados, no fue posible realizar un análisis al respecto ${ }^{13}$. En 2011 se publicó una revisión Cochrane sobre el uso de GTT en EM, que incluye dos estudios prospectivos doble ciego, aleatorizado y placebo-controlado. Los autores concluyen que la GTT es efectiva en el tratamiento de la EM. El único efecto adverso pesquisado es el riesgo de incrementar la hipoacusia ${ }^{14}$.

Las dosis utilizadas en EM varían según el estudio, de una hasta 12 inyecciones, con concentraciones que también varían, de $26 \mathrm{mg} / \mathrm{mL}$ hasta $80 \mathrm{mg} / \mathrm{mL}$. En nuestro caso utilizamos dosis de $40 \mathrm{mg} / \mathrm{mL}$ y un rango de una hasta 6 inyecciones. Además, el esquema utilizado también es variable y controversial, pero en nuestro caso utilizamos un esquema que separa en al menos un mes las inyecciones, dado que el efecto es retardado en muchas ocasiones $8,13,15$. En el algoritmo propuesto por el consenso internacional sobre el tratamiento de la EM, publicado el presente año, se incluye el uso de GTT en pacientes seleccionados. En nuestra experiencia, la GTT se utiliza luego del fracaso del control del vértigo con terapia médica, incluyendo el uso de dieta hiposódica, betahistina, diuréticos, corticoides, y derivación a psiquiatría ${ }^{16}$. Además, muchas veces los pacientes han realizado terapia de rehabilitación vestibular, sin respuesta.

La principal indicación de nuestra serie clínica es la EM, lo cual coincide con la literatura internacional. De acuerdo a la evidencia disponible, esta terapia estaría indicada en aquellos pacientes con vértigo refractario. Todos los pacientes que recibieron GTT habían recibido diversos tratamientos 
médicos, incluyendo antivertiginosos orales y corticoides transtimpánicos, con mala respuesta. La mejoría sintomática del grupo con EM se logró en el $100 \%$ de los casos, en el tiempo de seguimiento, siendo mayor que lo reportado previamente. Si bien, en nuestro caso no contamos con un registro adecuado, en la literatura se ha demostrado que además de mejorar el vértigo, el uso de GTT a largo plazo en EM, disminuye los niveles de ansiedad ${ }^{11}$.

Como se observa en la Tabla 1, tres pacientes tienen diagnóstico diferente a EM: hídrops retardado (HR), hídrops autoinmune y fístula perilinfática. La experiencia en estas entidades es limitada. En una serie con 10 pacientes sometidos a GTT, uno de ellos tenía el diagnóstico de hídrops retardado con sordera profunda del mismo lado, sin embargo, no se especifican los resultados para este paciente ${ }^{9}$. En pacientes con HR, tanto la terapia con corticoides transtimpánicos como con GTT, han sido utilizados en el control del vértigo con buena respuesta ${ }^{17}$. En nuestra casuística, el caso 7 con HR, a 2 meses de seguimiento presenta mejoría sustancial del vértigo. En el caso 4, debido a 6 intentos fallidos de GTT se decidió realizar laberintectomía quirúrgica, con buena respuesta. La laberintectomía quirúrgica ${ }^{18}$ se propone para casos refractarios a la GTT, pudiendo además ofrecer la colocación de un implante coclear y el aún en desarrollo implante vestibular ${ }^{19,20}$. En nuestra serie ofrecemos hemos sugerido laberintectomía quirúrgica luego de 6 inyecciones de GTT sin éxito sintomático.

El caso 8, con diagnóstico de fístula perilinfática posterior a timpanoplastía fue un caso desafiante, y dada la inexistente literatura al respecto, se decidió discutir con un panel de expertos en el comité de oídos de nuestra institución, quienes sugirieron la aplicación de gentamicina sobre la ventana redonda en pabellón, debido a la presencia de bridas e inflamación crónica que impiden la difusión de la gentamicina en el oído medio.

\section{Función vestibular}

La función vestibular debe ser evaluada para el seguimiento objetivo del tratamiento con GTT. Diversos protocolos incluyen la evaluación del nistagmo, prueba calórica, potenciales evocados miogénicos vestibulares (VEMP) y $\mathrm{VHIT}^{21-26}$. En la prueba calórica, se observa un aumento en la asimetría, que no siempre correlaciona con las variaciones en el vHIT. Esta variación, se mantiene independiente del número de dosis administradas ${ }^{21}$. La mejoría subjetiva del vértigo, correlaciona con una hipofunción vestibular mayor al 90\% medida por prueba calórica, posterior a la aplicación de GTT22. Por otro lado, Carey y cols. (2002) publicaron una serie de 17 pacientes con EM unilateral, que recibieron GTT. Las ganancias medias del VHIT, posterior al procedimiento, para los canales semicirculares (CSC) laterales, anteriores y posteriores, fue, respectivamente: 0,$40 ; 0,35 ;$ y 0,31 . Los autores de este trabajo sugieren que la GTT produce una lesión vestibular parcial, y además es importante resaltar que al lesionar un oído con GTT, al medir la ganancia del VHIT, aún persistirá la respuesta inhibitoria del canal coplanar al oído lesionado24. El año 2018 se publicó una revisión sistemática y metaanálisis, que incluyó cuatro estudios de pacientes con EM que recibieron GTT, incluyendo al de Carey y cols (2002). En el oído tratado, la ganancia de Ios CSC disminuyó en: 0,36 en el horizontal; 0,35 en el posterior y 0,28 en el superior. El estudio se realizó entre 12 a 94 días posterior al tratamiento. Los autores plantean que la disminución de la ganancia, particularmente para el CSC horizontal y superior, puede predecir el control del vértigo ${ }^{21}$. Un valor de ganancia para el CSC lateral mayor a 0,8 estaría asociado a la necesidad de administrar una segunda dosis de $\mathrm{GTT}^{23}$. Sin embargo, dado que al reducido número de pacientes evaluados con vHIT en los estudios publicados, y la falta de discriminación entre pacientes con dosis única 0 múltiples, aún no es posible establecer cuál es el valor de ganancia que se debe obtener como punto de corte, posterior al tratamiento.

Otro examen que puede ser utilizado en el seguimiento vestibular de estos pacientes, son Ios VEMPs, los que han mostrado estar ausente prácticamente en el $100 \%$ de los pacientes que reciben $\mathrm{GTT}^{9,25,26}$. En una serie de 10 pacientes con GTT, Ios VEMP cervicales (cVEMP) mostraron ausencia de potenciales en 9 de ellos. El único paciente con respuesta en el cVEMP, tenía además ausencia de respuesta con agua fría en la ENG, y presentó vértigo recurrente ${ }^{9}$. La falta de abolición de respuesta en cVEMP, podría considerarse como 
fracaso terapéutico, sin embargo, la experiencia publicada aún no es suficiente para establecer conclusiones al respecto. Se debe tener presente la limitación de este examen en caso de presentar ausencia de respuesta bilateral. No debe asumirse una ablación completa vestibular secundaria a la GTT en ausencia de respuesta en los VEMP, debido a que no evalúa el sistema vestibular completo.

\section{Cambios auditivos por GTT}

Es conocido el efecto ototóxico, con pérdida auditiva secundaria al uso de GTT en un rango que va de $0 \%$ a $90 \%$ de los pacientes ${ }^{27,28}$ y que puede llegar a pérdidas mayores a $30 \mathrm{~dB}$ en relación a los valores basales ${ }^{9,10,28}$. Como se observa en la Tabla 2; en dos de nuestros casos se constata disminución de la audición posterior a la GTT. Destaca el caso 2 , 6 y 9 (Tabla 2), que presentan una mejoría leve de la vía aérea posterior a la GTT. Este hallazgo, si bien es infrecuente, ya había sido descrito previamente en la literatura ${ }^{9,28,29}$. Dado el riesgo de hipoacusia posterior a la GTT no debiese indicarse en casos de enfermedad bilateral y siempre se debe advertir al paciente de este riesgo.

\section{Otros efectos}

Además de los cambios vestibulares y auditivos ya comentados, se ha reportado, como un efecto en extremo infrecuente del uso de GTT, la presencia de diplopia vertical binocular. Los pacientes comenzaron con esta sintomatología entre 4 a 8 días posterior a la GTT, presentando resolución espontánea dentro de 8 semanas $^{30}$. En nuestra serie no tenemos ningún paciente que haya reportado este efecto adverso.

\section{Limitaciones del estudio}

Este trabajo fue diseñado de forma retrospectiva, por lo que, hay datos relevantes que podrían haber resultado interesantes para el lector, que no pudieron ser rescatados de la ficha clínica. Creemos que resultaría de utilidad para generar más conocimiento sobre este tema, generar nuevos protocolos para el estudio prospectivo de estos pacientes, que reúnan un mayor número de pacientes, y que incluyan información estandarizada con más detalle.

\section{CONCLUSIONES}

Es importante seleccionar cuidadosamente a los pacientes que serán sometidos a tratamiento con GTT, siempre se deben agotar las instancias de manejo médico, incluyendo medicamentos y manejo por psiquiatría. La EM continúa siendo la principal indicación, pero existen otras patologías del oído interno que pudiesen beneficiarse de su uso. La experiencia adquirida en los últimos años nos permite recomendar el protocolo que separa en al menos un mes las inyecciones de GTT, por sus posibles efectos retardados y así disminuir la posibilidad de hipoacusia. El estudio vestibular $y$ auditivo de estos pacientes es fundamental y, actualmente debiera idealmente incluir audiometría, prueba calórica con video-oculografía y vHIT antes y después del tratamiento con gentamicina.

Tabla 2. Características auditivas y vestibulares, previo y posterior a gentamicina transtimpánica

\begin{tabular}{|ccccccc|}
\hline No & $\begin{array}{c}\text { PTP } \\
\text { pre (dB) }\end{array}$ & $\begin{array}{c}\text { PTP } \\
\text { pos (dB) }\end{array}$ & $\begin{array}{c}\text { Discriminación } \\
\text { pre (\%) }\end{array}$ & $\begin{array}{c}\text { Discriminación } \\
\text { pos }(\%)\end{array}$ & $\begin{array}{c}\text { Excitabilidad } \\
\text { pre }(\%)\end{array}$ & $\begin{array}{c}\text { Excitabilidad } \\
\text { pos }(\%)\end{array}$ \\
\hline 2 & 53 & 50 & 88 & 72 & 30,4 & 0 \\
3 & 32 & 63 & 100 & 72 & 50 & 50 \\
5 & 52 & 68 & 92 & 64 & 0 & 23 \\
6 & 73 & 68 & 72 & 80 & SR & SR \\
9 & 71 & 61 & 12 & 76 & & \\
\hline
\end{tabular}

SR = sin registro en ficha clínica. 


\section{BIBLIOGRAFIA}

1. McCall aA, Swan Ee, Borenstein JT, Sewell WF, Kujawa SG, McKenna MJ. Drug delivery for treatment of inner ear disease: current state of knowledge. Ear Hear 2010; 31: 156-65.

2. Hoffer Me, Balough BJ, Kopke RD, Henderson K, DeCicco M, Wester DC, O’leary MJ, Balaban C. Morphologic changes in the inner earof Chinchilla laniger after middle ear administration of gentamicin in a sustained-release vehicle. Otolaryngol Head Neck Surg 1999; 120: 643-8.

3. Lyford-Pike S, Vogelheim C, Chu E, Della Santina CC, Carey JP. Gentamicin is primarily localized in vestibular type I hair cells after intratympanic administration. J Assoc Res Otolaryngol 2007; 8: 497-508.

4. Hirvonen TP, Minor lB, Hullar te, Carey JP. Effects of intratympanic gentamicin on vestibular afferents and hair cells in the chinchilla. $J$ Neurophysiol 2005; 93: 643-55.

5. King EB, Shepherd RK, Brown DJ, Fallon JB. Gentamicin Applied to the Oval Window Suppresses Vestibular Function in Guinea Pigs. J Assoc Res Otolaryngol 2017; 18: 291-9.

6. Balough BJ, Hoffer ME, Wester D, O’Leary MJ, Brooker CR, Goto M. Kinetics of gentamicin uptake in the inner ear of Chinchilla langier after middle-ear administration in a sustained-release vehicle. Otolaryngol Head Neck Surg 1998; 119: 427-31.

7. Riveros h, Callejas C, Fernández F, Cohen M. Experiencia en el tratamiento de la enfermedad de Ménière con terapia transtimpánica. Rev Otorrinolaringol Cir Cabeza Cuello 2005; 65: 187-92.

8. Syed MI, llan 0, Nassar J, Rutka JA. Intratympanic therapy in Meniere's syndrome or disease: up to date evidence for clinical practice. Clin Otolaryngol 2015; 40: 682-90.

9. Celis-Aguilar E, Hinojosa-González R, ValesHidalgo 0, Coutinho-Toledo H. Refractory episodic vertigo: role of intratympanic gentamicin and vestibular evoked myogenic potentials. Braz J Otorhinolaryngol 2016; 82: 668-73.

10. Casani AP, Piaggi P, Cerchial N, Seccia V, FranceschinI SS, Dallan I. Intratympanic treatment of intractable unilateral Meniere disease: gentamicin or dexamethasone? A randomized controlled trial. Otolaryngol Head Neck Surg 2012; 146: 430-7.

11. Boleas-Aguirre MS, Sánchez-Ferrandiz N, GuillénGrima F, Perez N. Long-term disability of class A patients with Ménière's disease after treatment with intratympanic gentamicin. Laryngoscope 2007; 117: 1474-81.

12. Pereira N, Espinoza C, Concha R, Walker K. Enfermedad de Ménière: caso clínico y revisión de la literatura. Rev Hosp Clín Univ Chile 2008; 19: 166-75.

13. Cohen-Kerem R, Kisilevsky V, Einarson tR, Kozer E, Koren G, Rutka JA. Intratympanic gentamicin for Menière's disease: a metaanalysis. Laryngoscope 2004;114: 2085-91.

14. Pullens B, van Benthem PP. Intratympanic gentamicin for Ménière's disease or syndrome. Cochrane Database Syst Rev 2011; 16: CD008234.

15. Chia SH, Gamst AC, Anderson JP, Harris JP. Intratympanic gentamicin therapy for Ménière's disease: a meta-analysis. Otol Neurotol 2004; 25: 544-52.

16. Nevoux J, Barbara M, Dornhoffer J, Gibson W, Kitahara T, Darrouzet V. International consensus (ICON) on treatment of Ménière's disease. Eur Ann Otorhinolaryngol Head Neck Dis 2018; 135 : S29-S32.

17. Liu B, Zhang S, Leng Y, Zhou R, Liu J, Kong W. Intratympanic injection in delayed endolymphatic hydrops. Acta Otolaryngol 2015; 135: 1016-21.

18. Cabrera N, Dentone L, Stott C, Délano P. Laberintectomía quirúrgica. Rev Otorrinolaringol Cir Cabeza Cuello 2017; 77: 412-6.

19. Aedo-Sánchez C, Délano P. Implantes vestibulares. Rev Otorrinolaringol Cir Cabeza Cuello 2013; 73 : 271-5.

20. Alzérreca E, Pardo J, Délano P. Neuroprótesis en Otorrinolaringología: más allá del implante coclear. Rev Hosp Clín Univ Chile 2011; 22 : 318-24.

21. Marques PS, Días CC, Pérez-Fernández N, Spratley J. Instrumental head impulse test changes after intratympanic gentamicin for unilateral definite Ménière's disease: A systematic review and meta-analysis. Auris Nasus Larynx 2018; 45: 943-51.

22. Junet P, Karkas A, Dumas G, Quesada JL, Schmerber S. Vestibular results after intratympanic 
gentamicin therapy in disabling Menière's disease. Eur Arch Otorhinolaryngol 2016; 273: 3011-8.

23. Marques P, Manrique-Huarte R, Pérez-Fernández $N$. Single intratympanic gentamicin injection in Ménière's disease: VOR change and prognostic usefulness. Laryngoscope 2015; 125: 1915-20.

24. Carey JP, Minor lB, Peng GC, Della Santina CC, Cremer PD, Haslwanter T. Changes in the threedimensional angular vestibulo-ocular reflex following intratympanic gentamicin for Ménière's disease. J Assoc Res Otolaryngol 2002; 3: 43043.

25. Helling K, Schönfeld U, Clarke AH. Treatment of Ménière's disease by low-dosage intratympanic gentamicin application: effect on otolith function. Laryngoscope 2007; 117: 2244-50.

26. Ozluoglu LN, Akkuzu G, Ozgirgin N, Tarhan E. Reliability of the vestibular evoked myogenic potential test in assessing intratympanic gentamicin therapy in Meniere's disease. Acta Otolaryngol 2008; 128: 422-6.

27. Diamond C, O’Connell DA, Hornig JD, Liu R. Systematic review of intratympanic gentamicin in Meniere's disease. J Otolaryngol 2003; 32: 351-61.

28. Rah YC, Han JJ, Park J, ChOl BY, Koo JW. Management of intractable Ménière's disease after intratympanic injection of gentamicin. Laryngoscope 2015; 125: 972-8.

29. Wu IC, Minor LB. Long-term hearing outcome in patients receiving intratympanic gentamicin for Ménière's disease. Laryngoscope 2003; 113: 815-20.

30. Dresner SM, Kung NH, Palko JR, McJunkin JL, Goebel JA, Van Stavern GP. Skew Deviation and Partial Ocular Tilt Reaction Due to Intratympanic Gentamicin Injection, with Review of the Literature. Neuroophthalmology 2017; 41: 26870. 